\title{
Distribución de Aedes albopictus (Skuse) en Nuevo León, México, 2001-2004
}

\author{
Héctor $O$ rta-Pesina, $M$ en $C,{ }_{1}^{(1,2)}$ Roberto Mercado-Hernández, D $r$ en $C,{ }^{(2)}$
} José Fernando Elizondo-Leal, Biól.(1)

\section{Orta-Pesina H, Mercado-Hernández R, Elizondo-Leal JF. Distribución de Aedes albopictus (Skuse) en Nuevo León, México, 2001-2004. Salud Publica Mex 2005;47:163-165.}

\begin{abstract}
Resumen
Objetivo. Determinar la distribución de Aedes albopictus (Skuse) en N uevo León, México. Material y métodos Entre 2001 y 2004, utilizando métodos estandarizados de recolección de larvas de mosquitos, se analizaron 6371 muestras, de 167 localidades. Se estimaron proporciones de muestras positivas de Ae. albopictus, por municipio, por año, utilizando Excel. Resultados. Hubo 1179 muestras positivas para larvas de Ae. albopictus distribuidas en 10 municipios: cinco localizados en la llanura costera del Golfo de México, con clima semicálido, subhúmedo y con lluvias casi todo el año (Allende, Montemorelos, Linares, General Terán y Santiago); otros, Sabinas Hidalgo y Cadereyta, se localizan en las grandes llanuras del norte de A mérica, con clima cálido, semiseco y con lluvias poco frecuentes y, finalmente, A podaca, San N icolás y Escobedo en el área metropolitana de la ciudad de Monterrey. Conclusiones. Hasta noviembre de 2004, el segundo vector de mayor importancia epidemiológica en el ciclo de la transmisión de Ae. albopictus a escala mundial se encontró en 10 municipios del estado de Nuevo León.
\end{abstract}

Palabras clave: Aedes albopictus; Aedes aegypti; dengue; México

\author{
Orta-Pesina H, Mercado-Hernández R, Elizondo-Leal JF. \\ Distribution of Aedes albopictus (Skuse) \\ in Nuevo Leon, México, 2001-2004. \\ Salud Publica Mex 2005;47:163-165.
}

\begin{abstract}
A bstract
Objective. To determine the distribution of Aedes albopictus (Skuse) in N uevo Leon between 2001 and 2004. Material and Methods. Standardized methods were used to collect 6371 samples of mosquito's larvae from 167 sites. The proportions of samples positive for Ae. albopictus were obtained by municipality and year, using the software program Excel. Results. There were 1179 samples positive for Ae. albopictus. This species was distributed over 10 municipalities, as follows: Five on the Gulf of M exico C oastal Plains, which predominantly have a semi-warm, sub-humid climate, and precipitations almost year round (Allende, Montemorelos, Linares, General Teran, and Santiago); two (Sabinas Hidalgo, and C adereyta) localized on the N orth American $G$ reat Plains, which have a warm, semiarid climate, and scarce precipitations. The final three were A podaca, San N icolas, and Escobedo distributed over the metropolitan area of Monterrey C ity. Conclusions. Up to January 2004, the second most important epidemiological vector in the transmission cycle of Ae. albopictus worldwide was found in ten municipalities of N uevo Leon State, Mexico.
\end{abstract}

Key words: Aedes albopictus; Aedes aegypti; dengue; Mexico a invasión de Aedes albopictus aumenta el riesgo de introducción de nuevos serotipos del virus del dengue en el estado de Nuevo León, dada su capacidad de transmitir de modo vertical y horizontal a los distintos serotipos del virus. El Ae. albopictus está consi- derado como un vector de menor importancia que el Ae. aegypti en la transmisión del virus del dengue en áreas donde ambas especies son simpátricas. ${ }^{1}$

No obstante está considerado como el vector primario en áreas donde el Ae. aegypti está ausente. ${ }^{2-4}$ Se ha

(1) Laboratorio Estatal de Salud Pública en N uevo León, México.

(2) Laboratorio de Entomología Médica de la Facultad de Ciencias Biológicas, Universidad Autónoma de N uevo León, México.

Fecha de recibido: 17 de febrero de 2004 • Fecha de aprobado: 9 de febrero de 2005

Solicitud de sobretiros: Héctor O rta. Laboratorio Estatal de Salud Pública. Serafín Peña 2211, coloniaValle de la Silla, Ciudad Guadalupe, N uevo León, México.

Correo electrónico: romercad@ ccr.dsi.uanl.mx 
demostrado que el Ae. albopictus es capaz de transmitir transováricamente los cuatro serotipos del dengue..$^{5} \mathrm{~A}$. albopictus se localizó por vez primera en el municipio de Linares, en el estado de Nuevo León, en 1993; sin embargo, fue hasta 1997 cuando de 195 muestras colectadas por el personal del Departamento de Control de Vectores de la Secretaría de Salud en el Estado, en el municipio de Allende, y enviadas al Laboratorio Estatal para su identificación, 53 (27.2\%) se identificaron como Ae. albopictus. ${ }^{6}$

El propósito del presente trabajo fue determinar la distribución de Aedes albopictus en el estado de Nuevo León, México, entre los años 2001 y 2004, por su posible implicación en materia de salud pública en el estado.

\section{Material y métodos}

Utilizando métodos estandarizados de recolección de larvas para mosquitos del género Aedes, el personal de campo del Departamento de Control de Vectores de la Secretaría de Salud del estado de Nuevo León obtuvo las muestras, que se preservaron en alcohol a $70 \%$ y se enviaron al Laboratorio Estatal para su identificación; se emplearon claves para larvas de cuarto estadio de mosquitos de Norteamérica, ${ }^{7}$ y se usaron un microscopio estereoscópico y uno bacteriológico, con un aumento de 40X.

Se estimaron las proporciones de muestras positivas, por municipio, en cada año estudiado, utilizando la hoja de cálculo de Excel.

\section{Resultados}

El cuadro I destaca la distribución de muestras recibidas y positivas a Ae. albopictus, por año, municipio y localidad, de 2001 a 2004.

De un total de 1620 muestras recibidas por el Laboratorio Estatal de Salud, en 2001, 88 fueron positivas a la presencia de Ae. albopictus y 81 (92.05\%) correspondieron al municipio de Allende. En el municipio de Santiago se encontraron cinco muestras positivas $(5.67 \%)$, a finales del mes de septiembre, en recipientes que contenían agua de lluvias; una, en el municipio de General Terán a principios del mes de noviembre, también en un cacharro con agua de lluvia, y a principios del mes de julio, en el municipio de Linares, se localizó una muestra positiva en un tambo.

Durante 2002, de 1556 muestras, 268 manifestaron presencia del culícido. En el municipio de Allende se agregaron 18 nuevas localidades con 178 muestras positivas que corresponden a $66.42 \%$ del total en ese año. En el municipio de Santiago se encontraron 67 muestras positivas a Ae. Albopictus, que constituyen

\section{Cuadro I \\ Distribución de muestras positivas para Ae. ALBO PICTUS, POR MUNICIPIO Y POR AÑo. NUEVO LEÓN, MÉXICO, 2001-2004}

\begin{tabular}{llrcc} 
Año & Municipio & Localidades & $\begin{array}{c}\text { Muestras } \\
\text { positivas }\end{array}$ & $\begin{array}{c}\text { Porcentaje } \\
\text { por municipio }\end{array}$ \\
2001 & Allende & 21 & 81 & 92.05 \\
\hline$(1620)$ & Linares & 1 & 1 & 1.14 \\
\hline & Santiago & 3 & 5 & 5.67 \\
\hline & General Terán & 1 & 1 & 1.14 \\
\hline & Total & 26 & 88 & 100.00
\end{tabular}

\begin{tabular}{llrrc}
2002 & Allende & 18 & 178 & 66.42 \\
\hline$(1556)$ & Santiago & 14 & 67 & 25.00 \\
\hline & General Terán & 3 & 14 & 5.22 \\
\hline Montemorelos & 4 & 9 & 36 \\
\hline Total & 39 & 268 & 100.00
\end{tabular}

\begin{tabular}{llrrr}
2003 & Allende & 13 & 285 & 38.41 \\
\hline$(2698)$ & Santiago & 29 & 300 & 40.43 \\
\hline General Terán & 9 & 18 & 2.43 \\
\hline Montemorelos & 21 & 114 & 15.36 \\
\hline Sabinas Hidalgo & 22 & 24 & 3.23 \\
\hline Apodaca & 1 & 1 & 0.14 \\
\hline Total & 95 & 742 & 100.00
\end{tabular}

\begin{tabular}{llrrr}
2004 & Cadereyta & 2 & 25 & 30.86 \\
\hline$(497)$ & Escobedo & 1 & 18 & 22.22 \\
\hline Santiago & 1 & 23 & 28.39 \\
\hline San N icolás & 3 & 15 & 18.53 \\
\hline Total & 7 & 81 & 100.00 \\
& Totales & 167 & 1179 &
\end{tabular}

$25 \%$ del total. En General Terán se agregan tres localidades con 14 muestras positivas; en el municipio de Montemorelos, cuatro, y nueve muestras fueron positivas.

Para el año 2003 se recibieron 2698 muestras, de las cuales 742 fueron positivas. En Allende se observó una disminución del porcentaje de muestras positivas comparando con los años anteriores, mientras que en Santiago el porcentaje fue en aumento. Cabe señalar que en este último se encuentra el paraje turístico natural conocido como La Cola de Caballo, cascada de agua enclavada en la Sierra Madre Oriental con 800 msnm, y en la zona turístico-comercial de artesanos de Los Cavazos a orillas de la presa Rodrigo Gómez, ambas localidades muy frecuentemente visitadas por una gran cantidad de turismo nacional e internacional. En General Terán se muestrearon nueve localidades y el panteón, 18 muestras fueron positivas. En Montemorelos, que tiene 21 localidades, hubo 114 positivas (15.36\%). 
El municipio de Sabinas Hidalgo está dividido en áreas geoestadísticas básicas (AGEB), en 22 se determinaron 24 muestras positivas (3.23\%) para Ae. albopictus. En el municipio de Apodaca, perteneciente al área metropolitana de la Ciudad de Monterrey, con nueve municipios, se encontró una muestra positiva durante agosto en un cacharro con agua de lluvia.

Durante 2004 se recibieron 497 muestras, de las cuales 81 resultaron positivas para Ae. Albopictus; se destacan tres nuevos municipios: uno al Oriente de Monterrey (Cadereyta) y los dos restantes en el área metropolitana de la Ciudad de Monterrey (Escobedo y San Nicolás). En Cadereyta hubo 25 muestras positivas (dos localidades), mientras que en Escobedo, en una localidad hubo 18 positivas (22.22\%); por su parte, en San Nicolás se muestrearon tres localidades, y 15 fueron positivas (18.53\%). Nuevamente en Santiago se notificaron 23 muestras positivas (una localidad), que correspondieron a $28.39 \%$ del total para ese año.

En la figura 1 se presenta la distribución actual de Ae. albopictus en el estado de Nuevo León, México.

\section{Discusión}

El "mosquito tigre" Ae. albopictus ha demostrado una gran adaptabilidad a los diferentes climas que hay en Nuevo León, principalmente en los municipios de Allende ${ }^{6}$ y Santiago, con mayor preferencia en este último por ser una zona montañosa, con clima semicálido, húmedo, y con lluvias casi todo el año, así como en los municipios de Sabinas Hidalgo y Cadereyta, donde el clima fue semiseco, cálido, y con lluvias poco frecuentes. El arribo del mosquito Ae. albopictus al área metropolitana de la ciudad de Monterrey trae consigo una infinidad de interrogantes respecto a su importancia en salud pública, puesto que allí se observa un gran flujo de personas por ser la ciudad más industrializada de México, con alto comercio y un turismo en aumento. Por ello, se pretende llevar a cabo un estudio de la distribución espacial y temporal de este mosquito empleando sistemas de información geográfica, ${ }^{8}$ así como su asociación con otros culícidos. ${ }^{9}$ En conclusión, hasta noviembre de 2004 se ha encontrado el segundo vector de mayor importancia epidemiológica a escala mundial en el ciclo de la transmisión de la enfermedad de la fiebre del dengue (Ae. albopictus), en 10 municipios del estado de Nuevo León, cinco de ellos localizados en la llanura costera del Golfo de México (Allende, Montemorelos, Linares, General Terán y Santiago); otros, Sabinas Hidalgo y Cadereyta, en las grandes llanuras del norte de América y, finalmente, Apodaca, San Nicolás y Escobedo, en el área metropolitana de la ciudad de Monterrey.

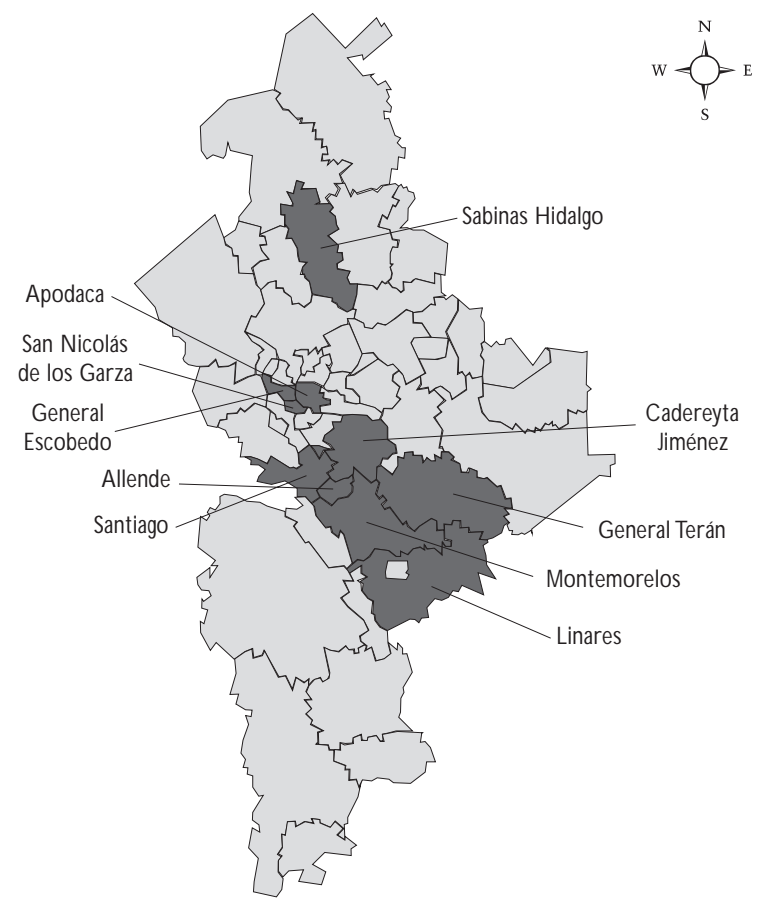

Figura 1. Distribución de Aedes albo pictus en el estAdo de Nuevo León, México, 2001-2004

\section{Referencias}

1. Chan YG, Ho BC, Chan KL. Aedes aegypti (L) and Ae. albocpictus (Skuse) in Singapore $\mathrm{C}$ ity. $\mathrm{O}$ bservation in relation to dengue haemorrhagic fever. Bull W HO 1971; 44:651-657.

2. Sabin AB. Research on dengue during W old W ar II.Am J Trop Med Hyg 1952;1:30-50.

3. Metsellar D, G rainger CR, 0 ei KG, Reynolds DG, Pudner M, Leake CJ et al. An outbreak of type 2 dengue fever in the Scychelles, probably transmitted by Aedes albopictus (Skuse). Bull W HO 1980; 58:937-943. 4. Q ui FH, Zhang, Shao L, Luo X, Yu Y. Studies on the rapid detection of dengue virus antigen by immunofluorence and radioimmunoassay. Chinese Med J 1981; 94:653-658.

5. Rosen L, Shroyer DA, Tesh RB, Frcier JE, Lien JC. Transovarial transmission of dengue viruses by mosquitoes; Aedes albopictus and Aedes aegypti. Am J Trop Med Hyg 1983; 32:1108-1119.

6. 0 rta-Pesina H, Mercado-Hernández R,Valdez-Rodríguez MA. Aedes albopictus in Allende City, N uevo León, México. J Am Mosq Control Assoc 2001; 17(4):260-261.

7. Darsie RF.The identification of Aedes albopictus in the $\mathrm{N}$ earctic region. J Am Mosq Control Assoc 1986; 2:336-340.

8. Mercado-Hernández R, Fernández-Salas I, Lozano-Fuentes S. Spatial analisis of dengue in Guadalupe, N uevo León, México, 1995-1996. Southwestern Entomol 2002;27(1):85-90.

9. Mercado-Hernández R,A guilar-G ueta J, Fernández-Salas I, Earl PR.The association of Aedes aegypti and Ae. albopictus in Allende, $\mathrm{N}$ uevo León, México. J Am Mosq Control Assoc 2005. En prensa. 